\title{
Reveal Hydrogen Behavior at Grain Boundaries in Fe-22Mn-0.6C TWIP Steel via In Situ Micropillar Compression Test
}

\author{
Xu Lu ${ }^{1}$. Dong Wang ${ }^{1} \cdot$ Di Wan $^{1} \cdot$ Xiaofei Guo ${ }^{2,3} \cdot$ Roy Johnsen $^{1}$
}

Received: 10 September 2021 / Revised: 5 November 2021 / Accepted: 19 November 2021

(c) The Author(s) 2022

\begin{abstract}
In this study, the effect of hydrogen on dislocation and twinning behavior along various grain boundaries in a high-manganese twinning-induced plasticity steel was investigated using an in situ micropillar compression test. The compressive stress in both elastic and plastic regimes was increased with the presence of hydrogen. Further investigation by transmission electron backscatter diffraction and scanning transmission electron microscope demonstrated that hydrogen promoted both dislocation multiplication and twin formation, which resulted in higher stress concentration at twin-twin and twin-grain boundary intersections.
\end{abstract}

Keywords Hydrogen $\cdot$ TWIP steel $\cdot$ Micropillar compression $\cdot$ Grain boundary $\cdot$ t-EBSD

\section{Introduction}

High-Mn twinning-induced plasticity (TWIP) steels with outstanding mechanical properties have drawn tremendous attention in the steel design and application realm [1,2]. On the one hand, the formation of twins can effectively suppress dislocation gliding, thus contributing to a pronounced dynamic strain hardening behavior. On the other hand, twin-twin intersections can act as stress concentrators inevitably causing microvoids formation and concomitant premature necking. Yet TWIP steel still shows excellent promise in the automotive industry due to its high strength and good ductility. Nevertheless, like other steels and alloys, such high-strength material suffers from hydrogen-induced

Available online at http://link.springer.com/journal/40195.

Dong Wang

dong.wang@ntnu.no

Xiaofei Guo

xiaofei_guo@shu.edu.cn

1 Department of Mechanical and Industrial Engineering, Norwegian University of Science and Technology, 7491 Trondheim, Norway

2 School of Materials Science and Engineering, Shanghai University, Shanghai 200444, China

3 Steel Institute (IEHK), RWTH Aachen University, 52072 Aachen, Germany degradation, which is typically referred to as hydrogen embrittlement. The mechanisms of hydrogen embrittlement have been under debate since this phenomenon was first reported by Johnson [3]. The most commonly acceptable mechanisms include hydrogen-enhanced localized plasticity (HELP) [4, 5], hydrogen-enhanced decohesion (HEDE) [6-8], hydrogen adsorption-induced dislocation emission (AIDE) [9-11], hydrogen-enhanced strain-induced vacancy (HESIV) [12] and the Defactant theory [13, 14]. The debate originates from many factors that may affect the hydrogeninduced crack initiation and propagation, such as the material's constituent and microstructure, hydrogen adsorption and diffusion, hydrogen content and distribution, and mechanical loading conditions $[15,16]$. Hence, it is pervasive to have several mechanisms working synergistically. Among, the synergistic effect of HELP and HEDE has been critically evaluated and quantified by summarizing both experimental and modeling results that involve both mechanisms [17, 18]. Typically, the hydrogen-enhanced dislocation activities at the crack tip further promote hydrogen accumulation, which could provoke the decohesion process simultaneously. The interaction and transition between each mechanism should be critically evaluated by combining the applied mechanical testing and hydrogen charging conditions.

Up to date, tremendous efforts have been dedicated to exploring the hydrogen embrittlement behavior in TWIP steels focusing on the effects of initial microstructure [19-24], alloying elements [25-30], strain rates [31, 32], 
and hydrogen charging methods [33-35]. Specifically, grain refinement is reported to effectively delay hydrogen-induced fracture by suppressing deformation twinning and reducing diffusible hydrogen fraction at grain boundary (GB) [19, 20, 36]. Moreover, it has been proposed that the addition of $\mathrm{Al}$ and $\mathrm{Cu}$ reduces the hydrogen embrittlement susceptibility in high-Mn steels [26, 27, 30], while Mn, Si and P show controversial effects [30,37, 38]. Additionally, a lower strain rate can cause pronounced hydrogen embrittlement by enhancing the twin-slip interactions [31]. Besides the aforementioned factors, the formation of intergranular fracture has been widely reported in TWIP steel under hydrogen attack [39-41]. It was demonstrated in a Fe-22Mn-0.6C TWIP steel that low angle grain boundaries (LAGBs) exhibit higher resistance to hydrogen-induced cracking compared to high angle grain boundaries (HAGBs) [41]. Beyond that, slow strain rate tests on the same TWIP steel revealed that hydrogen promotes twinning multiplications within grains having tensile axis orientations close to $\langle 111\rangle$ and $\langle 112\rangle / /$ rolling directions [40]. In contrast, it was further claimed that intergranular fracture primarily formed at locations with higher hydrogen concentration, while the relatively low hydrogen content regions do not necessarily contribute to the intergranular cracking [39].

All the above intergranular cracking behaviors were discussed based on the tensile tests on polycrystalline TWIP samples, which is hard to reveal the hydrogen-interrelated dislocation and twinning behavior at a specific type of GB. Therefore, it is necessary to design an elaborated small-scale test including only well-defined GBs. Among, micropillar (ranging from micro to sub-micro) compression test has been testified as an efficient method to reveal the local deformation behavior [42, 43]. Also, it is practicable to include one or more GBs in one micropillar using the focused ion beam (FIB) milling technique. It was demonstrated on bicrystalline (BC) micropillars in pure copper that a specially designed HAGB which allows dislocation slip transmission exhibited similar deformation behavior as single crystalline pillars [44]. By contrast, an arbitrary HAGB has been proven to be an effective barrier to dislocations and shows a pronounced hardening [45]. Meanwhile, micropillars containing coherent twin boundaries showed no strengthening effect compared to the single crystalline micropillars [45].

To reveal the hydrogen effect on the deformation behavior of micropillars at a small-scale level, a special experimental setup should be employed. Indeed, it is challenging to confine hydrogen in such a small volume of material, which needs both elaborated hydrogen charging and testing methods. On top of that, in situ charging is more appropriate than ex situ charging to avoid hydrogen degassing during the compression test. It has been demonstrated by the authors that nanoindentation coupled with a miniaturized three-electrode electrochemical charging cell is capable of performing both micropillar compression test and in situ hydrogen charging simultaneously [46]. A recent study investigated the effect of grain orientations on the hydrogen embrittlement behavior of high-Mn TWIP steel by compressing precharged micropillars and showed that hydrogen can increase flow stress of all the textured components [47]. However, GB was not involved in the above study.

Therefore, this study aims to deeply investigate the hydrogen effect on the dislocation and twinning behavior at specific HAGBs and LAGBs by performing an in situ BC micropillar compression test. Afterward, a thorough characterization combining scanning electron microscopy (SEM), transmission electron backscatter diffraction (t-EBSD) and scanning transmission electron microscopy (STEM) was carried out on the deformed micropillars to reveal the interaction between hydrogen and defects.

\section{Experimental}

\subsection{Material and Sample Preparation}

In this study, a Fe-22Mn-0.6C (wt\%) TWIP steel was investigated with the chemical composition as listed in Table 1. The steel was cast, hot rolled, and cold rolled to a thickness of $1.0 \mathrm{~mm}$. Subsequently, grain growth annealing was performed at $1150{ }^{\circ} \mathrm{C}$ in argon atmosphere for $5 \mathrm{~h}$. Afterward, disk specimens with a diameter of $12 \mathrm{~mm}$ were prepared via electrical discharge machining. The surface preparation was carried out in a sequence of mechanical grinding and polishing till a $40 \mathrm{~nm}$ colloidal silica suspension level. To select the optimum GBs, SEM coupled with an EBSD detector was utilized on the sample surface with micro-indent markings. The microstructure of steel is presented in Sect. 3.1. In this work, random GBs including both HAGB and LAGB were chosen. The crystallographic information of the selected GBs is summarized in Table 2 in Sect. 3.

\subsection{Fabrication of BC Micropillars}

The BC micropillars were fabricated using FIB in a Helios NanoLab DualBeam instrument (Thermo Fisher Inc., the USA). The ion beam current ranges from $9.1 \mathrm{nA}$ to $90 \mathrm{pA}$ at
Table 1 Chemical composition of the studied TWIP steel

\begin{tabular}{llllllll}
\hline $\mathrm{C}$ & $\mathrm{Mn}$ & $\mathrm{Al}$ & $\mathrm{Nb}$ & $\mathrm{V}$ & $\mathrm{Ti}$ & $\mathrm{N}$ & $\mathrm{Fe}$ \\
\hline 0.63 & 22.60 & 0.008 & 0.03 & 0.108 & 0.03 & 0.016 & Bal. \\
\hline
\end{tabular}


Table 2 Crystallographic information of the selected GBs $((\varphi 1 \Phi \varphi 2)$ denotes Euler angles, $\alpha$ is the tilt angle between the sample surface and the $\mathrm{GB},[U V W]$ is the misorientation axis, and $\theta$ is the misorientation angle between adjacent grains)

\begin{tabular}{llllll}
\hline GB & $\begin{array}{l}\text { Grain A } \\
(\varphi 1 \Phi \varphi 2)\end{array}$ & Grain B & {$[U V W]$} & $\theta$ & $\alpha$ \\
\hline LAGB1 & $(270.289 .2179 .6)$ & $(86.891 .32 .2)$ & {$[-1-105]$} & $4.2^{\circ}$ & $90^{\circ}$ \\
LAGB2 & $(355.74 .5301 .3)$ & $(291.692 .5181 .4)$ & {$[-11715]$} & $7.8^{\circ}$ & $50.1^{\circ}$ \\
HAGB1 & $(263.782 .5176 .0)$ & $(134.232 .471 .1)$ & {$[-121714]$} & $43.4^{\circ}$ & $89.2^{\circ}$ \\
HAGB2 & $(46.066 .6340 .4)$ & $(318.5133 .9185 .4)$ & {$[-7-411]$} & $33.5^{\circ}$ & $87.1^{\circ}$ \\
HAGB3 & $(316.893 .2142 .6)$ & $(75.672 .7315 .3)$ & {$[-8-65]$} & $52.3^{\circ}$ & $76.9^{\circ}$ \\
\hline
\end{tabular}

an accelerating voltage of $30 \mathrm{kV}$. To reduce the surface damage by Ga ion, a relatively low ion beam current at $90 \mathrm{pA}$ was utilized as the final step. The pristine micropillars have an average surface diameter of $2.14 \mu \mathrm{m}$ with a tapering angle of $\sim 2.8^{\circ}$. The aspect ratio (height to diameter) was designed to be 2.05 to prevent buckling during straining [48].

\subsection{In Situ Micropillar Compression Test}

The in situ micropillar compression test was carried out in the Hysitron TI950 TriboIndenter embedded with a miniaturized electrochemical charging cell aiming at in situ hydrogen charging. Details of the charging cell can be found in previous studies by the authors [46, 49]. The applied electrolyte is a glycerol-based borax solution diluted with $20 \%$ distilled water. $0.002 \mathrm{~mol} / \mathrm{L} \mathrm{Na}_{2} \mathrm{~S}_{2} \mathrm{O}_{3}$ was added to promote hydrogen adsorption. This electrolyte has been proven to prevent the sample surface from corrosion throughout smallscale mechanical testing [46, 50, 51]. Before the compression test, the sample was electrochemically charged at room temperature with a current density of $-1.7 \mathrm{~mA} / \mathrm{cm}^{2}$ for $5 \mathrm{~h}$ to guarantee a saturation of hydrogen in the micropillars [46]. The hydrogen charging condition was kept the same during the compression process to avoid hydrogen outgassing.

The micro-compression test was carried out by a roundflat-punch tip with $5.5 \mu \mathrm{m}$ of diameter. The micropillars were compressed under displacement-control mode with a displacement rate of $20 \mathrm{~nm} / \mathrm{s}$ up to $400 \mathrm{~nm}$, which was followed by a $1 \mathrm{~s}$ holding segment before unloading. In addition, cyclic loading in only elastic regime was performed to investigate the effect of hydrogen on the elastic behavior. At least six micropillars were compressed in each GB to check the reproducibility. During the compression test, the electrolyte was refreshed every $30 \mathrm{~min}$ to reduce the disturbance from hydrogen bubbles on the surface. The load-displacement curves were recorded to calculate the engineering stress and strain using the average half-height area and height. It needs to mention that the height of the pillar differs in adjacent grains due to a crystallographic orientationinduced channeling effect during FIB milling. Thus, the average height is typically adopted in the strain calculation.

\subsection{Postmortem Analysis}

The microstructure of micropillars after compression was characterized using SEM. Further, to investigate the deformation behavior along the GBs, target micropillars were lifted-out and welded on a copper grid. Lamellae with thickness $\sim 100 \mathrm{~nm}$ for high-resolution t-EBSD were obtained by FIB thinning. T-EBSD was carried out at $30 \mathrm{kV}$ with a step size of $20 \mathrm{~nm}$. Additionally, STEM was applied to reveal dislocation activities along the GB.

\section{Results}

\subsection{Microstructure of TWIP Steel and BC Micropillars}

The secondary electron imaging and normal direction inverse pole figure (ND-IPF) map of the studied material show a pure face-centered cubic (FCC) phase containing equiaxed grains with an average grain size of $87 \mu \mathrm{m}$ (Fig. 1a, b). The initial microstructure contains extremely low dislocation density from previous electron channeling contrast imaging (ECCI) observation [49]. The EBSD data analysis enables precise determination of the crystallographic relation between adjacent grains. For example, a HAGB with a misorientation of $57.3^{\circ}$ was selected and marked by the yellow arrows in Fig. 1a, b. Correspondingly, SEM images of pristine micropillars fabricated at the HAGB are presented in Fig. 1c, d. In this study, random GBs with both low and high misorientations were selected. A summary of detailed crystallographic information is provided in Table 2. The tilt angle $\alpha$ between the sample surface and the GB was precisely measured before the fabrication of pillars by cutting a trench at the end of the selected GB. This procedure is important for determining the location of micropillars during fabrication to guarantee pure compressive stress at the GB. In case $\alpha$ far deviates from $90^{\circ}$, the location of GB during milling should be offset from the central line to maintain the whole GB through the pillar. 
(a)
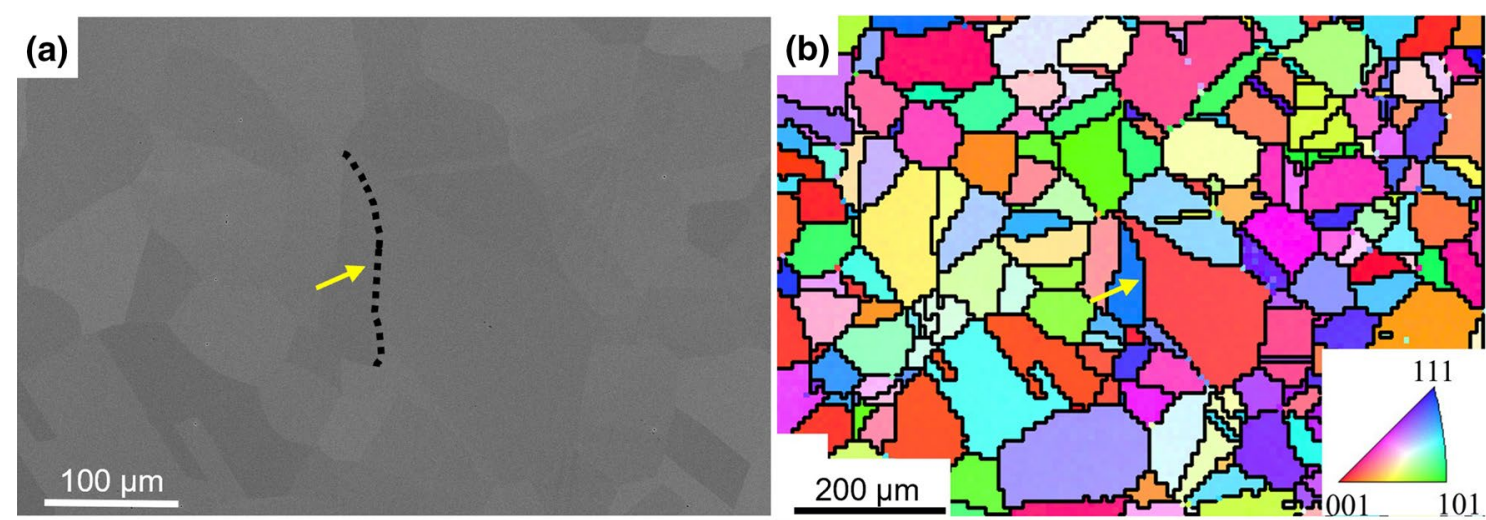

(c)

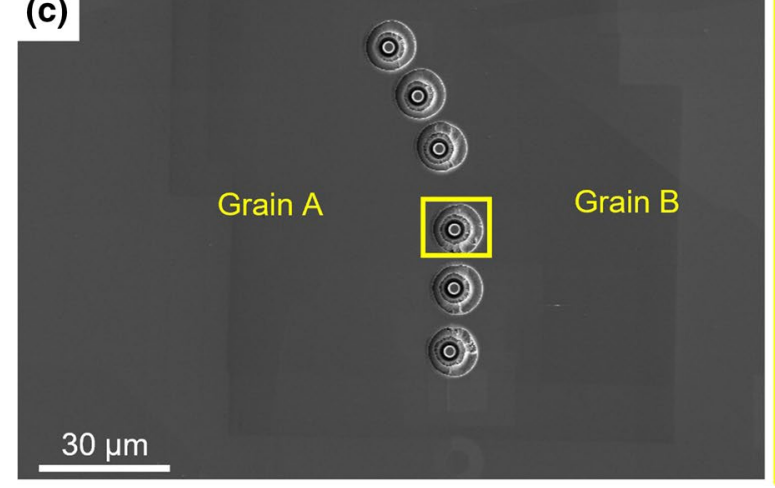

(d)

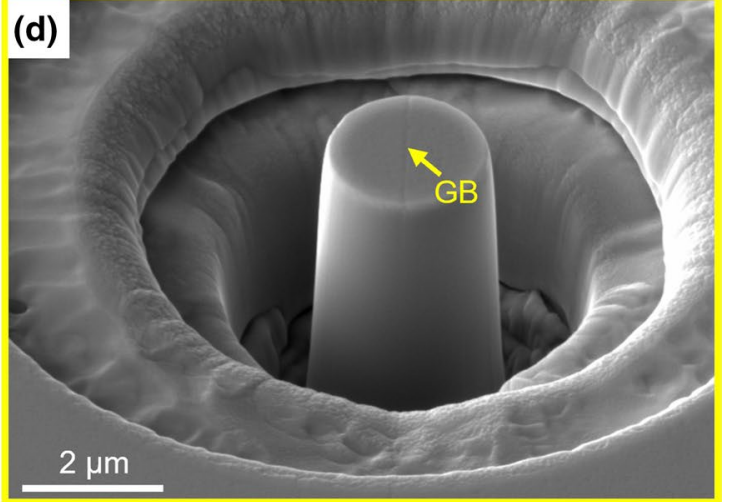

Fig. 1 a Microstructure of the studied TWIP steel showing the selected GB for micropillar fabrication; $\mathbf{b}$ the corresponding inverse pole figure (IPF) map; $\mathbf{c}$ SEM image of FIB-milled micropillars along the GB marked in $\mathbf{a}$; $\mathbf{d}$ higher magnification of the pristine micropillar enclosed by the yellow box in $\mathbf{c}$

\subsection{Hydrogen Effect on the Mechanical Properties of Micropillars}

Figure 2a presents the representative engineering stress-strain curves of the micropillars that were compressed in both hydrogen-free (air) and hydrogen-charged $(\mathrm{H})$ conditions at LAGB2. A pronounced stress elevation by hydrogen was observed, which is in accord with the previously reported results by the authors [46]. Specifically, in the air condition, the average yielding and maximum stresses are 290.2 $\mathrm{MPa}$ and 420.43 $\mathrm{MPa}$, which increase to 466.19 $\mathrm{MPa}$ and $551.05 \mathrm{MPa}$ under the $\mathrm{H}$ condition. Instead of small strain
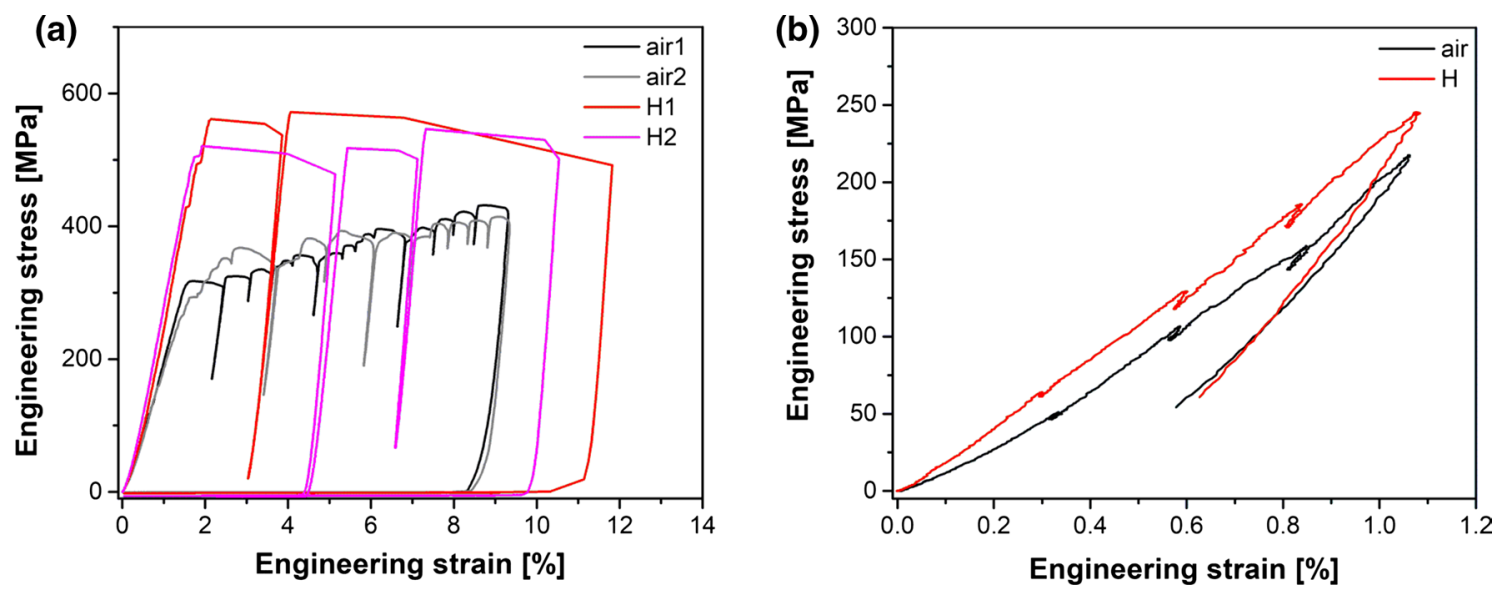

Fig. 2 Representative a engineering stress-strain curves; b stress-strain curves in the elastic regime for micropillars at LAGB2 that were tested in both hydrogen-free and hydrogen-charged conditions 
bursts observed in air condition, larger and fewer strain bursts were recorded on the hydrogenated micropillars. The strain burst in the TWIP steel micropillars can be considered as a result of either jammed dislocation reconstruction or twin formation. Furthermore, due to the sudden drastic plastic deformation that happened in the hydrogen condition, the indenter tip was unable to follow the deformed sample in the applied displacement control mode, and thus, the engineering stress after strain bursts reached to zero. As a comparison, in the air condition, the stress after strain bursts shows a smaller drop. In addition, micropillar compression test in the elastic region was carried out to investigate the hydrogen effect on the elastic behavior, and the representative stress-strain curves are presented in Fig. 2b. It shows higher elastic stress in the hydrogen-charged condition compared to the hydrogen-free condition. This phenomenon is reproducible in all tested GBs. It needs to be mentioned that all the compression tests were performed after the charging cell was installed. This means that no uninstallation and re-installation of the cell during the experiment. The re-installation of the sample after hydrogen

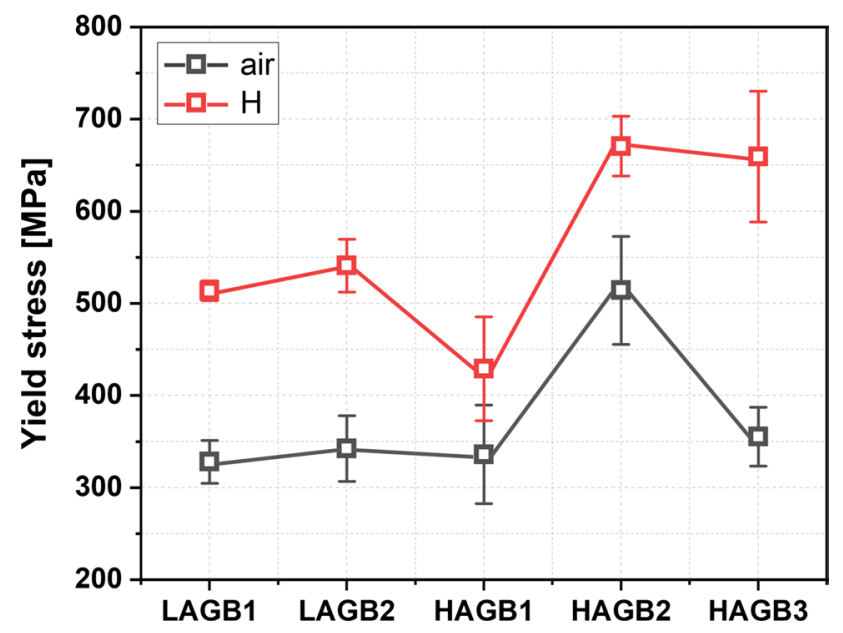

Fig. 3 Effect of hydrogen on the ultimate strength $\left(\sigma_{y}\right)$ for the tested micropillars

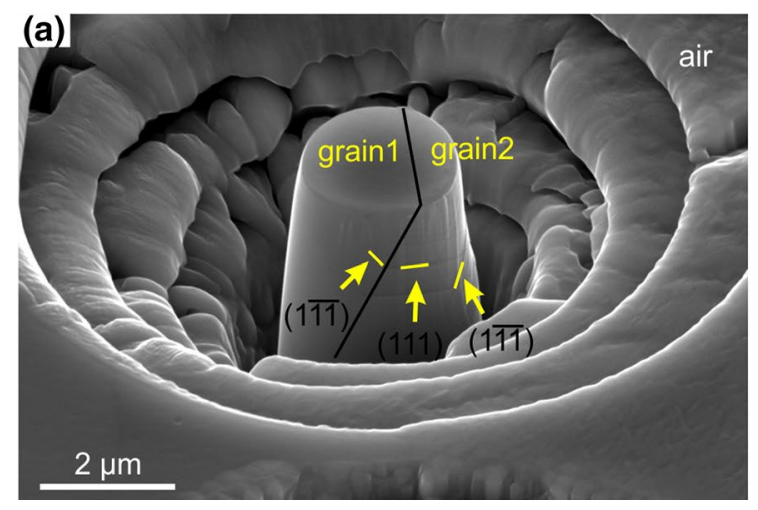

pre-charging typically results in a misalignment of the sample surface and further causes fake deviation of the elastic property compared to the air condition. Therefore, in the current study, in situ test would intrinsically remove this concern and what we observed is the pure effect from hydrogen.

The average yield strength $\sigma_{\mathrm{y}}$ with standard deviation in different testing conditions is summarized in Fig. 3. The $\sigma_{\mathrm{y}}$ is defined as the stress of the first large strain burst $(\geq 0.2 \%$ plastic strain), following criteria of $0.2 \%$ offset plastic strain in typical tensile testing. In all types of BC micropillars, an augment of $\sigma_{\mathrm{y}}$ is observed under hydrogen charging condition. In addition, all the GBs show approximately over $150 \mathrm{MPa}$ difference of $\sigma_{\mathrm{y}}$ in each testing condition. Beyond that, the strength of HAGBs was not necessarily higher than that of the LAGBs indicating that the crystallographic orientation relation in the active slip systems and twinning behavior in adjacent grains play a key role in determining the strength of pillars apart from misorientations.

\subsection{Hydrogen Effect on Deformation Behavior of Micropillars}

Figure 4 compares the representative SEM images taken from HAGB3 micropillars that were compressed in hydrogen-free and hydrogen-charged conditions. In the hydrogenfree condition, surface steps can be observed indicating the on-set of dislocation slip. It is found that $(1 \overline{1} \overline{1})$ slip plane is active in grain1, (111) and $(1 \overline{1} \overline{1})$ in grain2 (yellow lines). Unfortunately, the surface morphology in the hydrogencharged condition is non-detectable due to the corrosion product on the surface. In fact, the used electrolyte is aimed to preserve the surface integrity after surface charging, and it has been successfully applied on several different alloys [46, 50-52]. However, the studied TWIP steel is prone to corrosion, and the enhanced roughness induced by $\mathrm{Ga}$ ion during the FIB milling process can cause corrosion products. The corrosion products were caused by the deposition

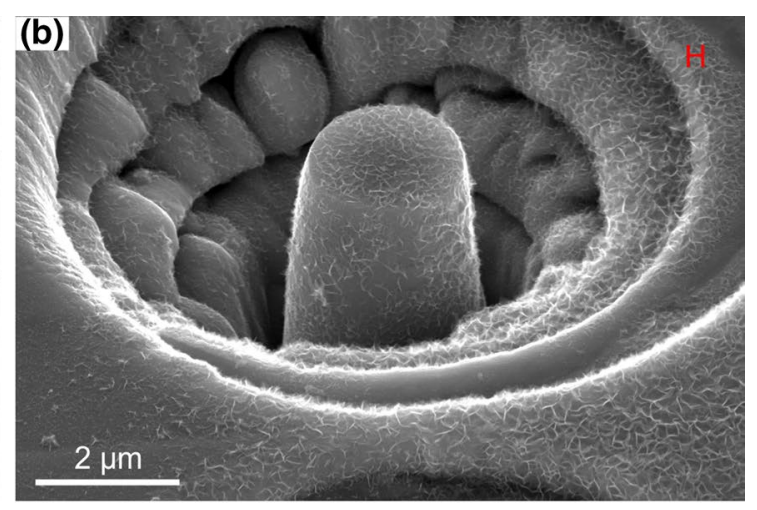

Fig. 4 Micrographs of deformed micropillars at HAGB3 that were tested in a hydrogen-free, $\mathbf{b}$ hydrogen-charged conditions 
process of organic impurities on the pillar surfaces either during the electrochemical charging process or post-cleaning, as indicated by the hairy lines on the surface (Fig. 4b). The observed corrosion products have been proven neither affecting the sample roughness nor influencing the mechanical properties. To reveal dislocations and twinning behavior at the GBs, the micropillars were lifted-out and welded on a copper grid for further characterization (Fig. 5a, b). FIB thinning was carried out at various ion beam currents until a thickness of $\sim 100 \mathrm{~nm}$ was reached (Fig. $5 \mathrm{c}$ ).

Afterward, t-EBSD and STEM were employed on the TWIP lamellae to disclose the deformation behavior intuitively and precisely at GBs. Figure 6 compares the t-EBSD results in HAGB3 micropillars compressed in hydrogen-free and hydrogen-charged conditions. It shows that the GB is tilting at $76.9^{\circ}$ (marked in Fig. 6a3). The image quality (IQ) map in air condition gives a somewhat vague strain contrast, which makes the identification of the active slip plane difficult (Fig. 6a3). However, the IQ map in hydrogencharged condition provides a superior strain contrast, which makes the determination of slip planes more straightforward (Fig. 6b3). Similar to the slip observation shown in Fig. 4a, one and two slip planes are active in grain 1 and grain2, respectively. Notably, evident strain contrast at the GB in the hydrogen-charged condition demonstrates a more pronounced plastic deformation. Additionally, strain concentration along the GBs indicates chaos of dislocation and twinning activities.
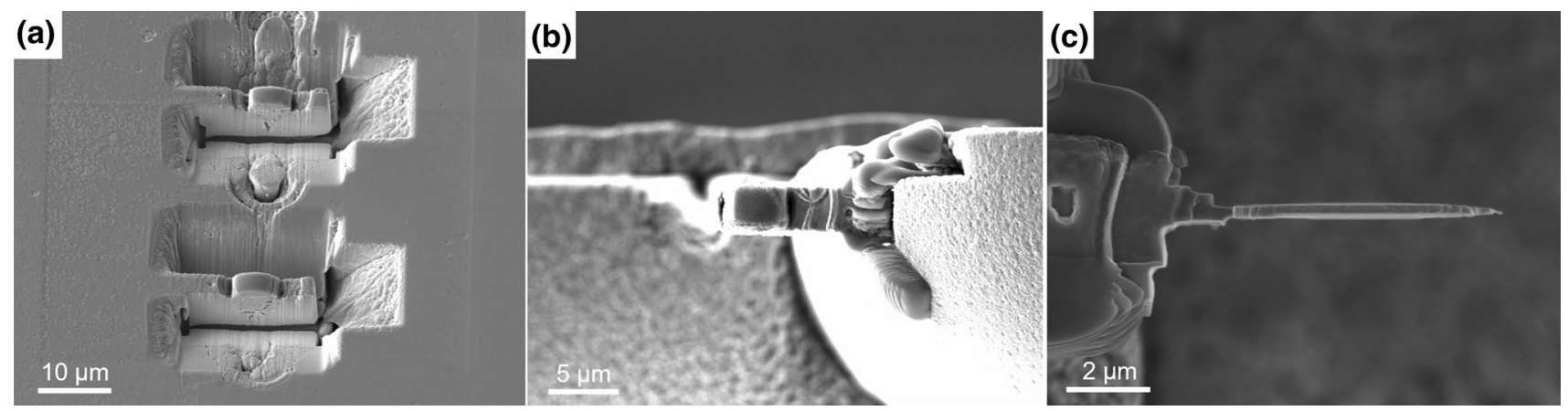

Fig. 5 a, b Lift-out procedures for the selected micropillars; $\mathbf{c}$ the milled lamella for t-EBSD and STEM characterization
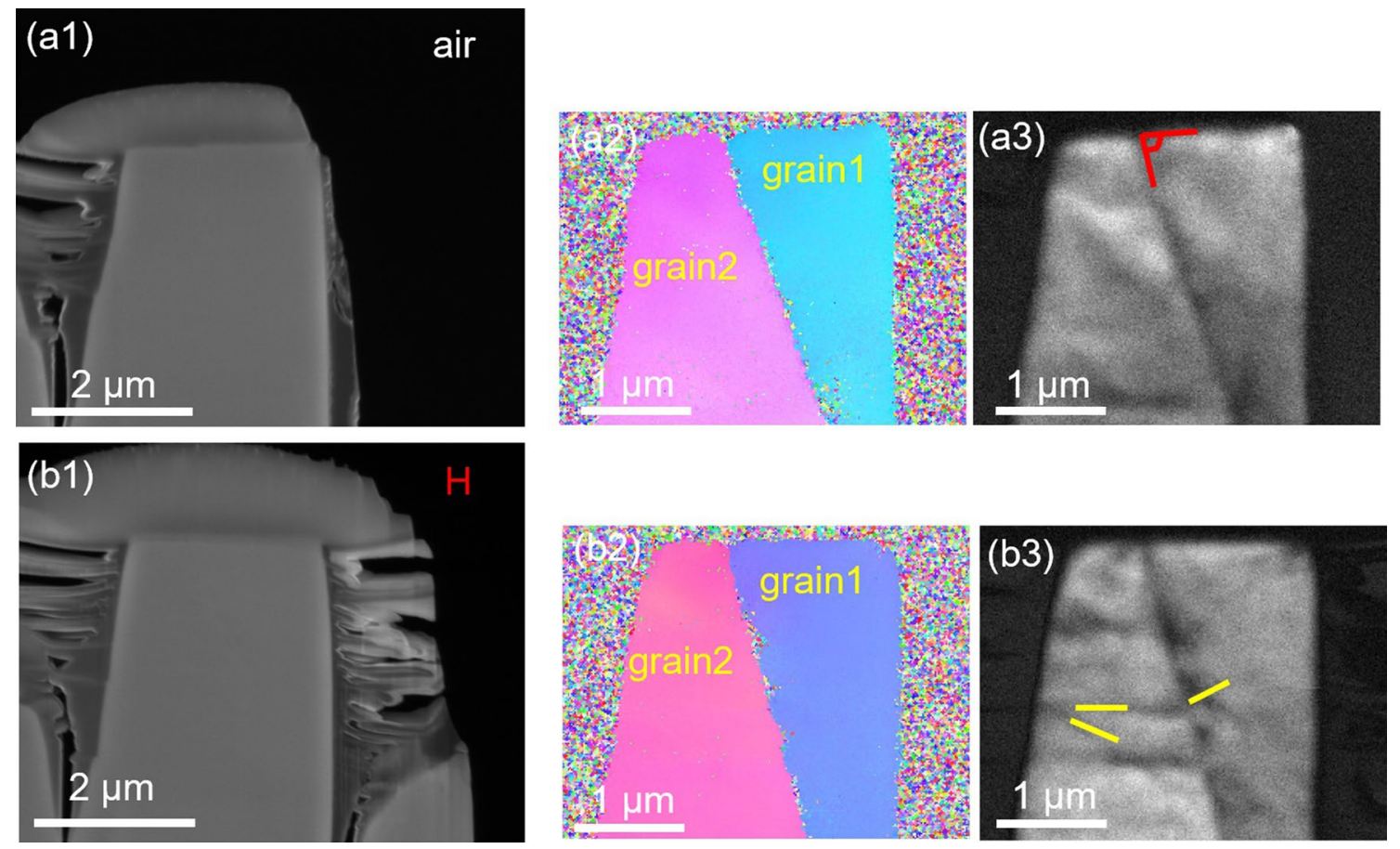

Fig. 6 Micrographs of the micropillar lamellae containing HAGB3 after compressed in a1 air, b1 hydrogen-charged conditions; and the corresponding t-EBSD results showing $\mathbf{a} 2, \mathbf{b 2}$ IPF, $\mathbf{a 3}, \mathbf{b 3}$ image quality (IQ) maps 
Parallelly, the bright-field STEM image in Fig. 7a demonstrates a weak dislocation activity and less strain concentration at the GB in the air case. Though hard to observe, few twins are identified (marked by yellow arrows), and dislocations are randomly distributed in the grain interior (marked by red arrows). The deformation is relatively homogeneous in each grain. In hydrogen-charged condition, however, twinning is clearly revealed (Fig. 7b). Deformation-twinning bands impinge the GB. As a result, high strain concentration at the intersections between twinning bands and the GB appears as dark contrast in the bright-field image, which is in accord with the t-EBSD result shown in Fig. 6b3. Moreover, in grain2, twin intersections on (111) and $(1 \overline{1} \overline{1})$ planes act as stress concentrators to raise strong strain contrast (marked by red circles in Fig. 7b). In addition, more deformation twins were formed, and abundant dislocations were observed close to the GB.

\section{Discussion}

\subsection{Hydrogen Effect on the Mechanical Properties of BC Micropillars}

The presence of hydrogen strongly elevates the stress of BC micropillars on all tested GBs, as shown in Fig. 3. This result is consistent with the previously reported phenomenon on nickel-based alloy [46], where the strengthening effect attributes to the hydrogen-enhanced dislocation multiplication and friction in the matrix. In addition, the dislocation cross-slips are inhibited due to a higher cross-slip activation energy following the perspectives of the HELP mechanism, and thus, the succeeding dislocations are blocked by the precursive ones, and higher stress is expected to overcome the barrier for further plastic deformation. The hardening behavior of alloys due to hydrogen charging was also frequently reported in the studies where hydrogen content is high [16]. The current charging condition guaranteed a saturated hydrogen level in the micropillars in accord with the prerequisite for the hardening effect. Moreover, t-EBSD results showed that dislocation transmission through both LAGBs and HAGBs is suppressed by hydrogen, which was proposed as another possible strengthening mechanism [46]. Besides, the stress-strain curves in hydrogen-charged micropillars (Fig. 2a) present merely discrete and large strain bursts, compared to the continuous yet small strain bursts in the hydrogen-free case. Typically, in a microscale polycrystalline material, the jump in the stress-strain curve is caused by the internal dislocation avalanche [53]. And the dislocation avalanches are primarily limited by the GBs. In the current study, it is reasonable to speculate that the large load drop accompanied by pronounced plastic deformation in the hydrogen-charged condition is associated with the sudden dislocation avalanche at highly stressed areas and multiple twin formation during the compression test, which will be discussed in detail in Sect. 4.2.

Another point that needs to be addressed is the strain hardening behavior for each testing conditions. As shown in Fig. 2a, a pronounced strain hardening can be observed on the stress-strain curves for hydrogen-free condition, which could be attributed to dislocation-dislocation interactions or related to dislocation exhaustion theory [54]. In contrast, each stress-strain curve in hydrogen-charged condition demonstrates a reduced strain hardening rate, which shows a softening effect. The softening effect induced by hydrogen during the compression test could be caused by the enhanced slip planarity, which resulted in an easier slip
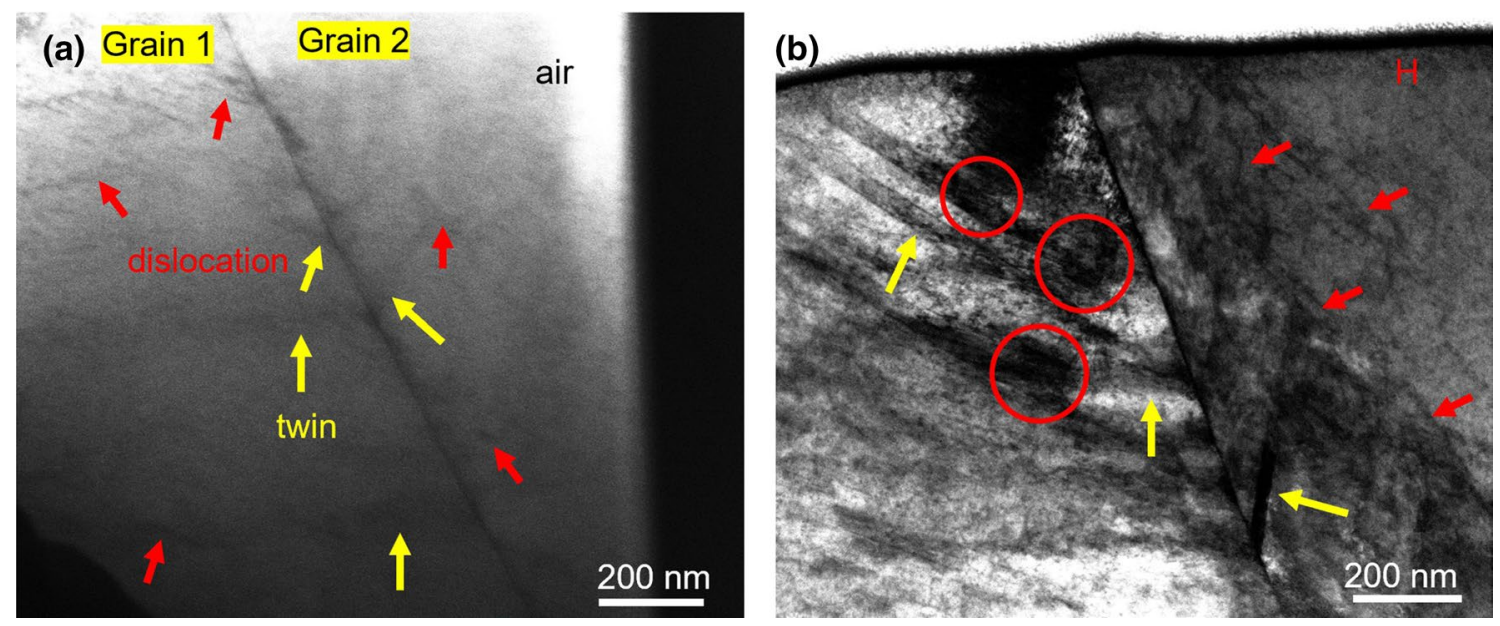

Fig. 7 Bright-field STEM images showing dislocations and twins in the deformed micropillars from HAGB3 tested in a hydrogen-free, $\mathbf{b}$ hydrogen-charged conditions. The yellow arrows refer to twins, and the red arrows refer to dislocations 
of dislocation out of the surface, in the framework of the HELP mechanism. Previous study on tungsten micropillars reported contradictory result by showing that a more pronounced hardening of tungsten was observed with the presence of deuterium [55]. The different results compared to the current study might be due to different strengthening mechanisms for FCC and BCC structures, which requires the support from advanced characterization techniques, such as high-resolution TEM.

In this study, both LAGBs and HAGBs were tested and showed a similar deformation trend. No clear difference was found in the stress elevation between HAGBs and LAGBs. The LAGB micropillars were claimed to show larger strain bursts in the hydrogen-free condition due to the low misorientation between adjacent grains, where dislocation is supposed to exhibit higher transmission through the GB. However, when hydrogen is introduced, LAGBs show likewise enhanced compressive stress and strain burst compared to those of HAGBs. In fact, it was clearly demonstrated from t-EBSD results in Ref. [46] that LAGBs can effectively suppress dislocation transmission.

Another interesting phenomenon in the stress-strain curve is that a hardening effect induced by hydrogen was observed in the elastic regime (Fig. 2b), which means an enhanced elastic modulus by hydrogen. In fact, the reduction of the material's elastic modulus with the presence of hydrogen was frequently documented [53, 56-59]. Specifically, the originally proposed HELP mechanism favors material softening and yield strength decrease due to hydrogen-enhanced dislocation mobility and dislocation slip [16]. The commonly reported reduction effect in body-centered cubic (BCC) iron links to the lowering of cohesive energy of the iron lattice by hydrogen [56, 60, 61]. Similarly, the same concept applied to the FCC polycrystalline pure $\mathrm{Ni}$ is revealed by the nanoindentation test [59]. Also, the degradation of Young's modulus in the $\langle 001\rangle$ oriented nickel single crystal is significantly affected by the vacancy clusters [62]. On the contrary, scarce studies mentioned the converse augment effect. To name a few, Young's modulus of BCC structured $\mathrm{Ta}, \mathrm{Nb}$ and $\mathrm{V}$ can be apparently elevated by a few atomic percent of hydrogen [63]. Analogically, the same trend was found in a ferritic steel [64]. Yet no one provided a detailed explanation for this behavior, and there is no documentation on the elastic property change by hydrogen in the FCC high-manganese steels.

In general, the elastic modulus reflects the force for atoms to vibrate from the equilibrium state, and it is proportional to the second derivative of the cohesive energy [63]. A higher value indicates that higher stress is needed to deviate an atom from its original lattice site. It is reasonable to speculate that in the highly hydrogenated micropillars, abundant hydrogen enters the lattice and occupies both octahedral and tetrahedral sites. The congested hydrogen expands the lattice structure and meanwhile increases the friction energy for atom vibration. Especially in the austenitic high-manganese steel, where hydrogen solubility is high. Actually, hydrogen-enhanced lattice friction has been reported on the same material [49]. Also, abundant hydrogen can bring in the solid solution hardening effect. However, the current result is contradictory to the concept of lowing cohesive energy by hydrogen. From the authors' viewpoint, the reduction of cohesive energy normally involves dislocation interactions at critical sites, such as sharp crack tips, where a higher stress level is expected. Moreover, the accumulation of sufficiently high concentrations of hydrogen is a prerequisite for triggering the decohesion mechanism. Since neither dislocation nor a locally higher amount of hydrogen is involved during elastic loading, the corresponding hydrogen effect on the cohesive energy should be further explored. Indeed, it is challenging to demonstrate and prove such speculation by merely experimental-based tests. Simulation methods like molecular dynamics are highly recommended to cooperate with the experimental approaches to thoroughly show the change of elastic behavior with the presence of hydrogen.

\subsection{Hydrogen Effect on Dislocation and Twining Activities at GBs}

Figures 6 and 7 clearly demonstrate the distinction in dislocation and twinning behavior at the GB. Compared to the micropillars that were compressed in hydrogen-free condition, pronounced dislocation interactions and deformation twinning were observed along the GB in the hydrogenated micropillars. On the one hand, hydrogen-enhanced dislocation multiplication and interaction have been well discussed in the previous studies [46, 49, 51]. According to the "Defactant" theory proposed by Kirchheim, the formation energy of dislocations was effectively reduced by hydrogen $[65,66]$. The enhanced interaction of multiple dislocations is one of the reasons for stress elevation. More recently, the influence of hydrogen on the dislocation structure is debated. Specifically, smaller dislocation cells and dense dislocation walls were reported in pure $\mathrm{Ni}$ under high-pressure torsion processing with the presence of hydrogen [67]. Following this work, the same dislocation structure change was later reported in ferritic-pearlitic low carbon steel during the fatigue-crack-growth test [68]. It was claimed that hydrogen enhances the microstructure evolution, which in this way gives rise to a strong work hardening and suppressed hydrogen redistribution within the spirit of the HELP mechanism. On the contrary, hydrogen induces larger dislocation cells termed as dislocation nanostructures, which attract hydrogen and act as local strain concentrators to initiate cracks [69]. In the current study, it was unable to probe dislocation cells on the micropillar lamellae in both cases, instead, dislocation tangling close to the GB proves the enhanced dislocation nucleation and 
multiplication induced by hydrogen (Fig. 7b).. On the other hand, hydrogen effectively promotes deformation twinning, as shown in Fig. 7b. A recent study demonstrated that hydrogen facilitates both twin multiplication and multiple twin system formation [40], which is partially consistent with the present results, although the formation of multiple twin systems was not observed in current work. In general, deformation twins formed from Shockley partial dislocations gliding on adjacent $\{111\}$ planes. They are planar defects comprising at least two contiguous stacking faults. Hydrogen was proven to reduce the stacking fault energy and promote stacking faults formation [70, 71], as proposed by both the HELP mechanism and the Defactant theory. Also, it was demonstrated in $\mathrm{Cu}-\mathrm{Al}$ alloys that twin lamella thickness decreases monotonically with a lower stacking fault energy [72]. Thus, twin density should be increased, and twin thickness should be decreased, which is supported by the experimental results in Ref. [73]. In the current study, hydrogen considerably promotes twin multiplication on the active $\{111\}$ planes (Fig. 7b), where a denser deformation twinning was formed. Indeed, by inhibiting twin formation, the hydrogen embrittlement resistance can be largely enhanced [47], since twin-twin and twin-GB intersections show higher strain concentration, and these areas are the critical sites for cracking [40, 41, 74].

Similar to dislocations, twins lamellae can trigger twin formation on the adjacent grain, appearing as if it is transmitted through the GB and forming adjoining twin pairs under continued straining [75]. Dislocation transmission through LAGB and HAGB was proven to be suppressed by hydrogen [46], yet this rule does not apply to the deformation twinning in this study. No twin transmission was found even in the hydrogenfree condition. In Fig. 7b, twins easily nucleate either from the GB or from the free surface, where defects reside. Instead of stimulating a twin on the other grain, the twins impinge the GB and raise strong stress concentration. Such stress concentrators can attract more hydrogen and are believed to promote cracking with further straining in the framework of HEDE mechanism. However, to study the effect of hydrogen on twin transmission and the cracking behavior, the micropillar compression test up to a higher strain level is recommended. Nevertheless, this study exploits the hydrogen effect on the deformation behavior close to LAGBs and HAGBs and provides valuable hints in understanding the hydrogen-assisted intergranular cracking behavior in the high-manganese steels.

\section{Conclusions}

As a summary, the effect of hydrogen on dislocation activity and twinning behavior at grain boundaries in a TWIP steel was investigated via a novel in situ micropillar compression test. The major conclusions are summarized as follows:
1. A bi-crystalline micropillar compression test was carried out on different LAGBs and HAGBs in hydrogen-free in situ hydrogen charging conditions. The stress-strain curves showed that the presence of hydrogen effectively increased the compressive stress in both elastic and plastic regimes. Hydrogen-enhanced lattice friction and dislocation multiplication are proposed as the main reasons.

2. An enhanced elastic modulus was observed in the hydrogen-charged condition, which could be due to increased friction energy for atom vibration.

3. t-EBSD results showed that dislocation transmission through both LAGBs and HAGBs was suppressed by hydrogen, which could be one strengthening mechanism induced by hydrogen.

4. No clear distinctions on mechanical property and microstructure change induced by hydrogen between LAGBs and HAGBs were observed.

5. Dislocation multiplication and twin formation are significantly enhanced by hydrogen, as revealed by the STEM result, leading to the formation of high stress concentrators at the GB, which are the potential crack initiation sites during plastic deformation.

Acknowledgements $\mathrm{X}$. Lu and D. Wang acknowledge the financial support from the Research Council of Norway through the project MHEAT (294689) and HyLINE (294739). X.F. Guo acknowledges the promotion of scientific exchange by the German Research Foundation SFB761 and the Start-up Foundation of Shanghai University.

Funding Open access funding provided by NTNU Norwegian University of Science and Technology (incl St. Olavs Hospital - Trondheim University Hospital).

\section{Declarations}

Conflict of interest The authors declare that they have no known competing financial interests or personal relationships that could have appeared to influence the work reported in this paper.

Open Access This article is licensed under a Creative Commons Attribution 4.0 International License, which permits use, sharing, adaptation, distribution and reproduction in any medium or format, as long as you give appropriate credit to the original author(s) and the source, provide a link to the Creative Commons licence, and indicate if changes were made. The images or other third party material in this article are included in the article's Creative Commons licence, unless indicated otherwise in a credit line to the material. If material is not included in the article's Creative Commons licence and your intended use is not permitted by statutory regulation or exceeds the permitted use, you will need to obtain permission directly from the copyright holder. To view a copy of this licence, visit http://creativecommons.org/licenses/by/4.0/.

\section{References}

[1] O. Grässel, L. Krüger, G. Frommeyer, L.W. Meyer, Int. J. Plast. 16, 1391 (2000) 
[2] B.C. De Cooman, Y. Estrin, S.K. Kim, Acta Mater. 142, 283 (2018)

[3] W.H. Johnson, Proc. R. Soc. Lond. 23, 168 (1875)

[4] H.K. Birnbaum, Mechanisms of Hydrogen Related Fracture of Metals (Illinois University at Urbana Department of Materials Science and Engineering, Urbana, 1989)

[5] H.K. Birnbaum, P. Sofronis, Mater. Sci. Eng. A 176, 191 (1994)

[6] W.W. Gerberich, R.A. Oriani, M.J. Lii, X. Chen, T. Foecke, Philos. Mag. A 63, 363 (1991)

[7] C.J. McMahon, Eng. Fract. Mech. 68, 773 (2001)

[8] L.B. Pfeil, Proc. R. Soc. Lond. 112, 182 (1926)

[9] S. Lynch, Corros. Rev. 30, 90 (2012)

[10] S.P. Lynch, Metallography 23, 147 (1989)

[11] S.P. Lynch, Scr. Mater. 61, 331 (2009)

[12] Y. Yao, X.L. Pang, K.W. Gao, Int. J. Hydrogen Energy 36, 5729 (2011)

[13] R. Kirchheim, Acta Mater. 55, 5129 (2007)

[14] R. Kirchheim, Acta Mater. 55, 5139 (2007)

[15] B. Sun, W. Lu, B. Gault, R. Ding, S.K. Makineni, D. Wan, C.H. Wu, H. Chen, D. Ponge, D. Raabe, Nat. Mater. 20, 1629 (2021)

[16] M.B. Djukic, G.M. Bakic, V. Sijacki Zeravcic, A. Sedmak, B. Rajicic, Eng. Fract. Mech. 216, 106528 (2019)

[17] M.B. Djukic, G.M. Bakic, V.S. Zeravcic, A. Sedmak, B. Rajicic, Corrosion 72, 943 (2016)

[18] M.B. Djukic, V. Sijacki Zeravcic, G.M. Bakic, A. Sedmak, B. Rajicic, Eng. Fail. Anal. 58, 485 (2015)

[19] N. Zan, H. Ding, X.F. Guo, Z.Y. Tang, W. Bleck, Int. J. Hydrogen Energy 40, 10687 (2015)

[20] Y. Bai, Y. Momotani, M.C. Chen, A. Shibata, N. Tsuji, Mater. Sci. Eng. A 651, 935 (2016)

[21] Y. Bai, Y.Z. Tian, S. Gao, A. Shibata, N. Tsuji, J. Mater. Res. 32, 4592 (2017)

[22] Y. Tomota, M. Strum, J.W. Morris, Metall. Mater. Trans. A 17, 537 (1986)

[23] Y. Kim, N. Kang, Y. Park, I. Choi, G. Kim, S. Kim, K. Cho, J. Korean Inst. Met. Mater. 46, 780 (2008)

[24] R.T. van Tol, L. Zhao, L. Bracke, P. Kommelt, J. Sietsma, Metall. Mater. Trans. A-Phys. Metall. Mater. Sci. 44, 4654 (2013)

[25] J.H. Ryu, S.K. Kim, C.S. Lee, D.W. Suh, H.K.D.H. Bhadeshia, Proc. R. Soc. A 469, 20120458 (2015)

[26] Y.S. Chun, K.T. Park, C.S. Lee, Scr. Mater. 66, 960 (2012)

[27] M. Koyama, E. Akiyama, K. Tsuzaki, ISIJ Int. 52, 2283 (2012)

[28] E.J. Song, H.K.D.H. Bhadeshia, D.W. Suh, Scr. Mater. 87, 9 (2014)

[29] D.K. Han, S.K. Lee, S.J. Noh, S.K. Kim, D.W. Suh, Scr. Mater. 99, 45 (2015)

[30] T. Dieudonne, L. Marchetti, M. Wery, J. Chene, C. Allely, P. Cugy, C.P. Scott, Corros. Sci. 82, 218 (2014)

[31] B. Bal, M. Koyama, G. Gerstein, H.J. Maier, K. Tsuzaki, Int. J. Hydrog. Energy 41, 15362 (2016)

[32] Y.J. Kwon, H.J. Seo, J.N. Kim, C.S. Lee, Corros. Sci. 142, 213 (2018)

[33] T. Michler, K. Berreth, J. Naumann, E. Sattler, Int. J. Hydrog. Energy 37, 3567 (2012)

[34] T. Michler, C. San Marchi, J. Naumann, S. Weber, M. Martin, Int. J. Hydrog. Energy 37, 16231 (2012)

[35] S.Y. Lee, B. Hwang, Korean J. Met. Mater. 55, 695 (2017)

[36] M. Koyama, E. Akiyama, Y.K. Lee, D. Raabe, K. Tsuzaki, Int. J. Hydrog. Energy 42, 12706 (2017)

[37] M. Koyama, S. Okazaki, T. Sawaguchi, K. Tsuzaki, Metall. Mater. Trans. A 47, 2656 (2016)

[38] S.M. Lee, I.J. Park, J.G. Jung, Y.K. Lee, Acta Mater. 103, 264 (2016)

[39] M. Koyama, E. Akiyama, K. Tsuzaki, Corros. Sci. 54, 1 (2012)

[40] X.F. Guo, S. Zaefferer, F. Archie, W. Bleck, Int. J. Miner. Metall. Mater. 28, 835 (2021)
[41] D. Wang, X. Lu, D. Wan, X.F. Guo, R. Johnsen, Mater. Sci. Eng. A 802, 140638 (2021)

[42] J.R. Greer, W.D. Nix, Appl. Phys. A Mater. 90, 203 (2008)

[43] D.M. Dimiduk, M.D. Uchic, T.A. Parthasarathy, Acta Mater. 53, 4065 (2005)

[44] N.V. Malyar, J.S. Micha, G. Dehm, C. Kirchlechner, Acta Mater. 129, 312 (2017)

[45] P.J. Imrich, C. Kirchlechner, C. Motz, G. Dehm, Acta Mater. 73, 240 (2014)

[46] X. Lu, D. Wang, J. Mater. Sci. Technol. 67, 243 (2021)

[47] D. Kim, G.H. Jang, T. Lee, C.S. Lee, Met. Mater. Int. 26, 1741 (2020)

[48] H. Zhang, B.E. Schuster, Q. Wei, K.T. Ramesh, Scr. Mater. 54, $181(2006)$

[49] D. Wang, X. Lu, Y. Deng, X. Guo, A. Barnoush, Acta Mater. 166, 618 (2019)

[50] D. Wang, X. Lu, Y. Deng, D. Wan, Z. Li, A. Barnoush, Intermetallics 114, 106605 (2019)

[51] X. Lu, D. Wang, Z. Li, Y. Deng, A. Barnoush, Mater. Sci. Eng. A 762, 138114 (2019)

[52] X. Lu, D. Wang, D. Wan, Z.B. Zhang, N. Kheradmand, A. Barnoush, Acta Mater. 179, 36 (2019)

[53] F.F. Csikor, C. Motz, D. Weygand, M. Zaiser, S. Zapperi, Science 318, 251 (2007)

[54] S.I. Rao, D.M. Dimiduk, T.A. Parthasarathy, M.D. Uchic, M. Tang, C. Woodward, Acta Mater. 56, 3245 (2008)

[55] X. Fang, M. Rasinski, A. Kreter, C. Kirchlechner, C. Linsmeier, G. Dehm, S. Brinckmann, Scr. Mater. 162, 132 (2019)

[56] R.E. Ricker, D.J. Pitchure, The influence of hydrogen on the elastic modulus and anelastic response of cold worked pure iron, Effects of Hydrogen on Materials, ASM Int (2009) pp. 219-226

[57] V.R. Skal's' Kyi, Z.T. Nazarchuk, S.I. Hirnyi, Mater. Sci. 48, 491 (2013)

[58] K. Tomatsu, K. Miyata, T. Omura, ISIJ Int. 56, 418 (2016)

[59] C. Muller, M. Zamanzade, C. Motz, Micromachines-Basel 10, 114 (2019)

[60] E. Lunarska, A. Zielinski, M. Smialowski, Acta Metall. 25, 305 (1977)

[61] M. Ortiz, J. Ovejero-Garcia, J. Mater. Sci. 27, 6777 (1992)

[62] G. Hachet, A. Metsue, A. Oudriss, X. Feaugas, Acta Mater. 148, 280 (2018)

[63] H.A. Wriedt, R.A. Oriani, Scr. Metall. Mater. 8, 203 (1974)

[64] C.D. Yin, J.J. Chen, D.D. Ye, Z. Xu, J.H. Ge, H.T. Zhou, Materials 13, $2263(2020)$

[65] R. Kirchheim, Scr. Mater. 62, 67 (2010)

[66] R. Kirchheim, Int. J. Mater. Res. 100, 483 (2009)

[67] S. Wang, A. Nagao, K. Edalati, Z. Horita, I.M. Robertson, Acta Mater. 135, 96 (2017)

[68] S. Wang, A. Nagao, P. Sofronis, I.M. Robertson, Acta Mater. 144, 164 (2018)

[69] P. Gong, J. Nutter, P.E.J. Rivera-Diaz-Del-Castillo, W.M. Rainforth, Sci. Adv. 6, 46 (2020)

[70] A.E. Pontini, J.D. Hermida, Scr. Mater. 37, 1831 (1997)

[71] X. Lu, Y. Ma, M. Zamanzade, Y. Deng, D. Wang, W. Bleck, W.W. Song, A. Barnoush, Int. J. Hydrog. Energy 44, 20545 (2019)

[72] Y. Zhang, N.R. Tao, K. Lu, Scr. Mater. 60, 211 (2009)

[73] C. Zhang, H.H. Zhi, S. Antonov, L. Chen, Y.J. Su, Scr. Mater. 190, $108(2021)$

[74] X.F. Guo, S. Zaefferer, F. Archie, W. Bleck, Procedia Struct. Integr. 13, 1453 (2018)

[75] M.A. Kumar, I.J. Beyerlein, R.J. McCabe, C.N. Tome, Nat. Commun. 7, $13826(2016)$ 